\title{
Le paradigme astral existe-t-il chez J.M.G. Le Clézio?
}

DOI: http://dx.doi.org/10.12775/LC.2020.025

Résumé: Les moments où le protagoniste masculin regarde les constellations (Le Chercheur d'or) ou le soleil (Le Procès-verbal) donnent-ils lieu à des méditations sur la lignée, le temps, la condition humaine? Existe-t-il un paradigme astral, soit un rêve de plasmaticité (Coccia) qui ne sépare pas en lieux bipolaires (la terre versus le ciel)? Nous faisons l'hypothèse d'un espace astral qui ne serait pas au-dessus et à part, à voir, ou à calculer mais qui est un souffle, une atmosphère où vivre au rythme cosmique. Comment ce paradigme astral remet-il en question le voir ou l'habiter? Quel enjeu, outre la centralité du soleil selon Copernic? Plusieurs stratégies mettent en mouvement ce qui fige la représentation du monde céleste. Lanalyse du rapport au ciel dans Le Chercheur d'or permet de saisir comment Alexis se défait d'une manière d'habiter pour devenir souffle. La matière de L'Inconnu sur la terre, dont la forme de l'essai poétique facilite une rêverie de la lévitation, assez classique, est précisément cette plasmabilité qui dépasse le rêve commun de voler, tel Icare, référence mythologique que Le Clézio fait jouer tout en la déconstruisant.

Mots clés: astres, mouvement, plasmabilité, Le Clézio

\section{Is There a Paradigme Astral in Le Clézio’s Novels?}

\begin{abstract}
Are the times when the male character looks up to the sky and contemplates the constellations (Le Chercheur d'or) or particularly the sun (Le Procès-verbal) a lead to genealogy, time or human condition reflexions? Is there a Paradigme astral, a dream of plasmaticité which ceases to split Le Clezio's work in two antagonists places, (earth versus sky)? We test the hypothesis of an astral area which would rather be a breathe, an atmosphere living to the cosmic rythm than a place up and

\footnotetext{
* Maître de conférences habilitée à diriger des recherches et commissaire d'exposition; membre de Textes \& cultures, Université d'Artois. Elle fut vice-présidente de l'association des lecteurs de J.M.G. Le Clézio pendant six ans, puis l'a présidée de 2013 à 2015 avant d'être rédactrice en chef de la revue.

E-mail: isabelle.rousselgillet@univ-artois.fr | ORCID: 0000-0003-2785-8675.
} 
apart, to see, or to calculate. How can this Paradigm question our view, our way to live in? What stake, besides the sun's centrality brought by Copernic? Various strategies set in motion what freezes the representation of the celestial world. The analysis of the connection to sky in Le chercheur d'or allows to understand how Alexis leaves his way of living in to become breath. The matter of L'Inconnu sur la terre, which, due to his poetic essai's form, simplifies a dream of levitation, rather mundane, classic, precisely is that plasmabilite which furthers the common dream to fly, just like Icare, mythological reference that Le Clézio stages while deconstructing it in the meantime.

Keywords: stars, mouvement, plasmabilité, Le Clézio

ifférents rapports au temps sont vécus au prisme d'un protagoniste masculin qui lève le regard sur les constellations ou sur le soleil dans les romans lecléziens. Nous faisons l'hypothèse qu'il existe un paradigme astral, un rêve de plasmaticité, qui ne découpe pas en lieux bipolaires (terre versus ciel) une œuvre souvent mise par la critique sous la coupe de dialogismes qui la structurent. Nous allons mettre à l'épreuve cette hypothèse d'un espace astral qui ne serait pas toujours au-dessus et à part, à voir, (le leurre du Chercheur d'or ${ }^{1}$ ) ou à calculer (la projection du géomètre Adam depuis sa lucarne dans Le Procès-verbal) mais une atmosphère, un souffle, où être au plus près du rythme cosmique. Autrement dit, si ciel (air) et terre (sol) sont souvent pensés en miroir ou de façon exclusive, certains passages romanesques ne remettent-ils pas en cause cette partition que viennent entretenir des médiateurs divers (axis mundi)? Quelles sont alors les implications d'une conception astrale de notre condition? Quel enjeu, outre la centralité du soleil, selon Copernic? Comment ce paradigme remet-il en question le voir et l'habiter? Relève-t-il d'une tentative pour déshabiter le monde?

\section{Présence des astres}

Étoiles, astres - lune comme soleil - abondent dans les récits de Jean-Marie Gustave Le Clézio. Leur fonction est souvent utilitaire comme repères pour les marins (Le Chercheur d'or, Raga). Leur présence atteste d'un goût pour la mer mais aussi pour l'astronomie, goût que J.M.G. Le Clézio aurait depuis l'enfance, nourri qu' il fut par la consultation des cartes et de livres comme L'Astronomie populaire de Camille Flammarion publié en 1879. Dans un article bien antérieur (Roussel-Gillet: 2008), nous nous étions penchées sur l'influence de ce livre sur l'imaginaire et l'écriture de J.M.G. Le Clézio. Toutefois, poursuivre l'inventaire des étoiles d'un récit à l'autre avec une volonté panoramique risque fort de ne saisir qu'une évolution à l'œuvre ou de conclure à la myriade car le panorama a pour implicite le fait de vouloir dominer du regard ou d'embrasser depuis un pivot. Cette épistémologie du regard risque fort de passer à côté de son objet, piégée

1 Les œuvres de J.M.G. Clézio citées ici sont toutes parues chez Gallimard, Paris: Le Procès-verbal (1963), Les Prophéties de Chilam Balam (1976), L'inconnu sur la terre (1978), Le Chercheur d'or (1985), Onitsha (1991). 
par le primat du regard qu'encourage toute une tradition anthropocentrée, renforcée par les instruments astronomiques comme la longue-vue. Il nous semble que c'est plutôt en analysant précisément comment les protagonistes regardent le ciel que notre objet d'étude devient davantage épistémologique.

Une remarque liminaire s'impose: la contemplation du ciel suppose souvent la perte de la verticalité pour les protagonistes masculins, Adam (Le Procès-verbal) ou Alexis (Le Chercheur d'or), qui, quoique marcheurs, ont un rapport à la jambe d'appui peu ancré. Le premier s'amenuise dans le sol pour s'y fondre, le second n'a de rapport à la terre-matière que dans les tranchées de la guerre en France, et il est bien souvent pris de vertiges à l'île Rodrigues. Tous deux s'allongent pour se tendre vers le ciel. Autant dire que l'humain perd un peu de sa superbe, de sa fameuse conquête de l'être debout. Ce constat ne relève pas simplement de l'ordre pratique, la posture horizontale facilite aussi une partition entre ciel et terre, comme si les deux n'étaient qu'en miroir. Le protagoniste se situerait en dessous d'une réalité qui le domine. L'Inconnu sur la terre, au titre emblématique, n'invite-t-il pas plutôt à reconsidérer la manière d'être sur la terre?

\section{Représentation(s)}

Pour éviter de figer le regard, il faudrait laisser agir le mouvement ; mais il existe des obstacles à ce mouvement. La représentation cartographique est le premier des six obstacles que nous relevons dans les écrits de J.M.G. Le Clézio.

[.... le re- du mot "représentation" n'est pas répétitif, mais intensif $[\ldots]$. La representatio est une présentation soulignée (appuyée dans son trait et/ou dans son adresse: destinée à un regard déterminé) $[\ldots]$ ( Nancy 2003: 73).

La représentation est bien une question de regard déterminant. Elle peut simplifier le ciel en un planisphère à un temps donné sans rotation ; les étoiles sont alors fixées comme la carte l'est, fixée au mur, faite image. La carte du ciel est bien d'abord une image, une représentation dans le bureau paternel au sein de la diégèse (Le Chercheur d'or) ou un document isolé en exergue (pour le lecteur, dans Raga). Ce document visuel encourage la tentation d'immobiliser le voir sur un objet. La carte est ni plus ni moins épinglée, la carte de l'île Rodrigues avec ses relevés, fixée "à côté du plan du ciel” (Le Clézio 1985: 63). "On immobilise l'objet du voir quand on le considère avant tout comme un texte à déchiffrer, une énigme à résoudre. Erwin Panofsky n'envisageait-il pas l'iconologie comme la discipline vouée, devant les images, à 'résoudre l'énigme du sphinx'? Mais n'est-ce pas simplifier l'image que de supposer en elle une 'clé' d'interprétation qui serait capable d'ouvrir toutes ses portes?" interroge Georges Didi-Huberman (2018: 20). C'est bien là toute la problématique du Chercheur d'or.

Une seconde manière de faire une fixation, est de s'arrimer à un souvenir ressassé, tel Alexis qui fixe toute étoile au souvenir de la première fois qu'il l'a vue, ce qui n'est pas sans poser la question d'une nostalgie, du spleen, d'une jouissance à ne pas se dégager du passé, d'une façon d'auréoler du passé ce qui advient au présent. Nous ne citerons qu'un passage 
où Alexis sur le pont du navire, récite et re-cite l'enfance, récitation qui devient le signe du déni d'un temps irréversible tout autant que sa preuve.

Dans le ciel, les étoiles brillent d'un éclat fixe. Je les regarde avec attention, je les cherche toutes, ce soir, comme si elles allaient me dire par leurs dessins les secrets de ma destinée. Le Scorpion, Orion et la silhouette légère du Petit Chariot. Près de l'horizon, le navire Argo avec sa voile étroite et sa longue poupe, le Petit Chien, la Licorne. Et surtout, ce soir, celles qui me font ressouvenir des belles nuits du Boucan, les sept feux des Pléiades, dont notre père nous avait fait apprendre par cœur les noms, que nous récitions avec Laure, comme les mots d'une formule magique: Alcyone, Electre, Maïa, Atlas, Taygète, Mérope ... Et la dernière, que nous nommions après une hésitation, si petite que nous n'étions pas sûrs de l'avoir vue: Pléïone. J'aime dire leurs noms encore aujourd'hui, à mi-voix, dans la solitude de la nuit, car c'est comme si je savais qu'elles apparaissaient là-bas, dans le ciel du Boucan, par la déchirure d'un nuage. (Le Clézio 1985: 159)

Le personnage reste géocentré en dépit de son souci des cieux. Rêveur, la tête dans les étoiles, il produit une équivalence entre ciel et terre qu'il met souvent en miroir. Le ciel, "comme si c'était de l'eau", "comme dans un miroir" (ibid.: 45) est un espace lisse. Cette mise en regard suscite d'ailleurs des correspondances dans d'autres arts que la littérature. Plusieurs œuvres d'Anselm Kiefer dédiées au médecin et astrologue anglais du XVI ${ }^{\mathrm{e}}$ siècle, Robert Fludd, sont, par exemple, inspirées de la théorisation des connexions entre macrocosme céleste et microcosme terrien, ce qui conduit Kiefer à voir un ciel étoilé dans les graines d'un tournesol. L'analogie est incessante dans les romans lecléziens, les lumières artificielles de la ville, la nuit, sont de façon attendue comparées à des "astres". Pourtant, quelques lignes de L'Inconnu sur la terre brouillent ce miroir mimétique, à sa place, une terreciel aquatique devient un espace symbiotique où tout se fond dans la comparaison: "l'air comme l'eau, comme du sable" (Le Clézio 1978: 143).

Une autre modalité de fixation est plastique, dessiner des étoiles sur une page les immobilise en image. Et il en est de ces étoiles qui s'égaillent dans les pages lecléziennes.

Mais c'est tout le ciel qui est immobilisé, ainsi dans toute la première partie de L'Inconnu sur la terre: "Le ciel ne bouge pas" (ibid.: 44). Il devient couleur: "et le ciel: grand, indécis ${ }^{2}$, où cesse toute perspective. Ciel gris, blanc, bleu." (ibid.: 110). Indécis, que nous relevons, est cependant l'indice, à mi-parcours du récit, d'un tremblement possible des lignes. Sinon “Le ciel est grand, et il n'y a rien d'écrit, rien à dire” (ibid.: 111). Est-ce à dire qu'il n'y a écriture qu'à condition de mouvement, de temps et de révolutions? S'affirme surtout chez J.M.G. Le Clézio la lumière, force vitale à l'état pur, et la présence de la mer, son miroir encore:

Peut-être que l'on devient pareil à l'eau et au ciel, lisses, sans pensée. Peut-être qu'on n'a plus ni raison, ni temps, ni lieu. Chaque jour est semblable à l'autre, chaque nuit se recommence. Dans le ciel nu, le soleil brûlant, les dessins figés des constellations. Le vent ne change pas: il souffle au nord, chassant le navire. (Le Cézio 1985: 165)

\footnotetext{
2 C'est nous qui soulignons en italique dans toutes les citations de ce texte.
} 
Une quatrième modalité est de faire paysage: "Tout était bien calme. Le ciel occupait les trois quarts du panorama. Et la terre, en dessous, était si paisible qu'on aurait dit - qu'elle continuait le ciel. Vous voyez le genre." (Le Clézio 1963: 247) Le paysage est alors défait de l'horizon si cher à Michel Collot mais non de tentation esthétique, voire d'abstraction, dont nous pouvons lire une variante plus géométrique, toujours dans Le Procès-verbal: "Les barreaux, au nombre de trois verticaux et de deux horizontaux, compartimentent un ciel pareil aux murs. C'était une division arbitraire, mais cependant harmonieuse, et dont le chiffre, douze, faisait bizarrement songer aux Maisons du Ciel selon Mamilius”. (ibid.: 258-259), “[... ] d'après Mamilius, la Huitième Maison du Ciel est celle de la mort” (ibid.: 259). Épinglage, jusqu'à la mort, donc.

Cependant, la manière la plus littéraire et, somme toute, la plus convenue est de figer dans La Référence Mythologique: Apollon dieu du soleil pour Adam, le jardin édénique biblique perdu ainsi que le mythe de la toison d'or et des Argonautes pour Alexis. Rappelons qu'Alexis a appris à regarder les étoiles avec son père, dont il tient aussi le rêve de trouver un trésor. Le mythe de Jason fortement mobilisé comme intertexte oriente dès lors le récit selon les modalités d'une quête d'un or qui brille au firmament, qui brille tant qu'il laisse en point aveugle la sœur, dénommée Laure (à entendre "l'or"), tout comme la compagne Ouma (mère-monde). Nous pensons alors ici à l'installation Inferno de Romeo Castellucci en 2008 E.T.O.I.L.E.S, inspirée de La Divine Comédie de Dante composée de trois grands poèmes: l'Enfer, le Purgatoire, le Paradis qui se conclut sur le même mot "stelle". Pour l'adaptation de l'Enfer pour le festival d'Avignon, Castellucci a placé en hauteur 5 téléviseurs: un écran pour chaque lettre du mot étoile. Il fait chuter le E, le S, le second $\mathrm{E}$ puis le L, laissant apparaître le mot TOI, dont les trois lettres tombent à leur tour pour exploser au sol. Qui serait ce TOI dans Le Chercheur d'or? TOI, mère dont Alexis entend trop la voix lisant la Bible, ou TOI, Ouma, qu'il entend si peu. La voix des pères, pourvoyeurs de repères, occulterait celle des femmes. Lire Le Chercheur d'or confronte à la surdité comme aux points aveugles. Et de fait, dans les premiers romans de J.M.G. Le Clézio, les aveuglés par le soleil sont nombreux: Adam, Besson, Hogan... À cette cécité s'ajoute une prédisposition psychologique: Alexis éprouve une sensation mélancolique qui l'enkyste dans le ressassement de la disparition - d'une maison, d'un être -, son travail de deuil restant inachevé. Une autre prédisposition est de projeter ses états d'âme, ce qui favorise des correspondances au spleen baudelairien, par exemple quand Alexis est soldat: "[...] le ciel au-dessus de nous est plombé, lourd. Les orages crèvent en averses brutales [...]” (Le Clézio 1985: 291). Sur l'axe temporel, Alexis entretient un rapport lacunaire au passé, qu'il retrouve par fragments comme autant de traces survivantes. Cela vaut aussi pour le passé collectif, ainsi du vaisseau Argo voué à être reconstruit pièce par pièce, définitivement non originel. Il y a une évidence de la perte, mais aussi un refus de la possibilité active de la perte: Alexis agit longtemps en archéologue qui se leurre dans la complaisance à l'origine. Il n'y aura pas résurrection possible, le passé est passé, la maison natale est détruite, les morts à la guerre sont morts. Mais cette expérience du ressassement est sa manière d'être dans le temps: en quête de traces (liées au pseudotrésor), dans une certaine perception de la durée (qui sera suspendue par l'insolation ou la guerre), de la mémoire (le lancinant souvenir) et du désir (le temps auprès d'Ouma). Sur l'axe spatial, si l'on dissocie artificiellement temps/espace, l'accent mis sur les correspondances donne une table de réorientation entre microcosme et macrocosme, "depuis 
la Renaissance sous sa modalité néoplatonicienne", et dans l'épistémé du XVI siècle pour garantir une "connaissance ordonnée" (Descola 2005: 359).

Enfin, il est une référence culturelle qui gèle encore plus dans le destin tracé, fixé: écrire la présence d'étoiles comme signe d'une prédestination qui rejoint la métaphysique médiévale. Le ciel est divinatoire dès la scène avec le père: "Peut-être que c'est écrit dans les étoiles, voilà ce que je pense, peut-être qu'il est écrit dans les étoiles comment il faudrait faire pour que rien ne change et que nous soyons sauvés." (Le Clézio 1985: 50) Et, une page plus loin, le mode hypothétique porté par les modalisateurs d'incertitude cède à l'affirmative ; les étoiles sont des "traits de feu qui tracent la destinée des hommes et permettent aux secrets de se réaliser" (ibid.: 51). C'est une des ritournelles d'Alexis qui s'accroche aux signes, des astres aux désastres provoqués par l'orage et la banqueroute, alors qu'il scelle lui-même son destin. Le mythe d'Icare qu'avait pointé Jennifer Waelti-Walters (1981) dans la structure imaginaire des premiers romans lecléziens impose aussi l'image d'un échec, par brûlure et chute, chute que l'on peut encore lire dans des œuvres ultérieures au Procès-verbal: les journées sont "brûlantes sous le soleil d'avril", comme "une chute dans un vide vertical" (1985: 204). Comme dans la nouvelle Cœur brûle, où il est aussi question de brûlure du soleil, d'échec et de chute: "Il avait fait si chaud l'été où Pervenche était partie. L'asphalte fondait dans les rues, les arbustes séchaient dans leurs pots. Le ciel était bas, il se mélangeait à la mer grise, une eau lourde, plombée, qui bougeait à peine, $[\ldots]$. Clémence se souvenait de ces journées, comme si la chaleur et la couleur du ciel et de la mer avaient joué un rôle déterminant dans la fuite de Pervenche, l'avaient conduite au désastre, à la destruction" (Le Clézio 2000: 15).

Pour poursuivre cette analyse du Chercheur d'or dans son rapport au temps, soulignons que germe l'idée d'échapper au destin, et d'accueillir autrement, au présent: "Il fait nuit à présent, j'entends jusqu'au fond de moi le bruit vivant de la mer qui arrive." (Le Clézio 1985: 375). L'évocation de la perte peut être promesse d'allégement et de liberté:

Maintenant, je sais que c'est ainsi qu'a fait le Corsaire après avoir retiré son trésor des cachettes du ravin, à l'Anse aux Anglais. Il a tout détruit, tout jeté à la mer. Ainsi, un jour, après avoir vécu tant de tueries et tant de gloires, il est revenu sur ses pas et il a défait ce qu'il avait créé, pour être enfin libre.

Sur la plage noire, je marche, dans la direction de la Tourelle, et je n'ai plus rien. (ibid.: 373)

Même si la melancholia peut reprendre le dessus, il y a là un défaire au sens que Martin Heidegger donne à ce mot, pour retrouver le "destinement" de l'être (Heidegger 1990: 203-204), non plus le destin inscrit aux astres, mais le destinement vers soi. S'individuer sans être le répétiteur du Corsaire ou des Argonautes serait la vraie transmutation alchimique vers soi, et non plus poursuivre un or mythique, forgé à la fabrique de l'imagination. La nuit, la mélancolie, le mirage du Corsaire sont autant d'ombres, au sens jungien (Jung 2014: 70), par lesquelles passe Alexis. Et il n'est qu'un adverbe de temps pour nous signifier ce passage réalisé vers cette conscience et vers soi: "maintenant", "à présent".

Néanmoins, cette révolution n'advient pas si facilement, les épiphanies et autres dérèglements du temps n'ont parfois aucune finalité, aucun lien avec la formation du personnage, si tant est qu'il en existe un dans certains livres, si l'on pense à L'Inconnu sur la terre. Dans plusieurs textes lecléziens, le temps se trouve suspendu dans un éternel inamovible, 
avec des références très explicites à un au-delà "vers ce pays d'où l'on ne revient pas" (Le Clézio 1978: 168) ou au vol comme "rêve d'immortalité" (1978: 205). "Ici c'est un peu de temps du ciel qui s'écoule. C'est un peu de temps sans limite, du temps qui ne morcelle plus, qui ne défait plus, qui ne flétrit plus. Un peu de temps qui vole à travers l'espace, oiseau immortel” (ibid.: 213). À renfort de leitmotive et d'anaphores, le temps est ralenti, suspendu, infini.

Le personnage lui-même s'immobilise, tel un buvard fixatif, ainsi Adam est-il d'emblée une surface absorbante: dans la diagonale du ciel, "le jaune le frappait en pleine face, mais sans se réverbérer [...] sans faire d'étincelles ni le moindre petit reflet" (Le Clézio 1963: 15-16). Il rappelle les croyances du passé (ou des Shadocks) et les peurs au point d'halluciner le soleil muni de tentacules ou devenu araignée d'or (ibid.: 23). Alors, "Un univers de terreurs enfantines: le ciel, vu du rectangle de la croisée, sembla prêt à se détacher et à s'abattre sur nos têtes" (ibid.: 23-24). À ces dérèglements et visions apocalyptiques, s'ajoute la prémonition. Et les deux extraits suivants tirés également des premières pages remettent en question l'héliocentrisme:

Il leur dirait un tas de choses, à ces enfants, quand le moment serait venu: il leur dirait, par exemple, que la terre n'est pas ronde, qu'elle est le centre de l'univers, et qu'ils sont le centre de tout sans exception. (1963: 19)

On leur dirait aussi qu'il faut n'avoir peur que d'une chose, que la terre se retourne, et qu'ils soient la tête en bas les pieds en l'air, et que le soleil tombe sur la plage, aux alentours de six heures, et fasse bouillir la mer, et éventre tous les petits poissons. (ibid.: 19-20)

Comment sortir de ces fixations aux accents prophétiques (Ook Chung 2001) sinon en rappelant l'impermanence des lueurs ou le rêve de lucioles, soit une manière d'être aérienne, plus volatile que géocentrée, comme celles des "êtres légers, dansants, visibles par instants comme de courtes flammes, puis disparaissant, hop hop!” (Le Clézio 1978: 145)

\section{Mouvement(s)}

Que l'image de la carte elle-même migre dans la transmission d'un père à son fils est bien insuffisant pour ne pas l'enkyster. Une métamorphose effective par le mouvement peut toutefois s'exercer à trois niveaux, de manière plus ou moins heureuse. La première concerne l'écriture avec le recours à la métaphore, toujours opératrice de déplacement, ou à une poésie de la liste qui traite les mots comme une matière sonore; ainsi de la liste d'étoiles différée dans L'Inconnu sur la terre jusqu'aux quasi dernières pages (ibid.: 302-304).

La seconde concerne le rapport entre le paysage et le personnage. La rêverie de l'envol suppose toujours une polarisation du bas vers le haut, mais aussi un tropisme qui ouvre un espace dilaté, à la symbolique analysée par Bachelard. Ce rêve de s'immerger dans les grands espaces est récurrent chez le narrateur: "Je voudrais vous parler loin, longtemps, avec des mots qui ne seraient pas seulement des mots, mais qui conduiraient jusqu'au ciel, jusqu'à l'espace, jusqu’à la mer" (ibid.: 7). Si échapper à la gravité ne dépolarise pas, l'action peut désubjectiviser, voire anonymiser. Ainsi d'un sourire d'enfant inconnu qui "reste dans 
le ciel" (1978: 8), ou d'une allusion globalisante aux "Peuple des nuages" (ibid.: 167) ou "peuples célestes" de Taos à Acoma (ibid.: 231) à propos desquels le narrateur n'hésite pas à évoquer une "illumination" (ibid.: 234).

Dans Le Chercheur d'or, les nombreuses aubes si privilégiées et leurs colorations rendent le ciel changeant, "Alors c'est comme si le temps faisait un bond en arrière, renversait son cours. Il y a quelques instants encore, c'était le soir, la lumière s'éteignait, mais un soir infini, qui conduisait au néant. Et maintenant, je vois qu'il est juste midi, le soleil est au zénith, et je sens sa chaleur et sa lumière sur mon visage et sur mes mains" (Le Clézio 1985: 82).

Ainsi, le personnage se met-il au diapason du cycle naturel, "De l'aube au crépuscule, je suis la marche du soleil dans le ciel, des collines solitaires de l'est jusqu'aux montagnes qui dominent le centre de l'île. Je vais à la manière du soleil, en arc de cercle, le pic sur l'épaule, mesurant au théodolite les accidents du terrain qui sont mes seuls points de repère. Je vois l'ombre des arbres girer lentement, s'allonger sur la terre" (ibid.: 204-205). La marche est féconde, mais force est de constater que c'est le regard qui prime, quoiqu'il passe par le regard de l'autre.

Dans le silence apaisé de l'été, avec le mugissement lointain des brisants, les dessins des constellations sont des légendes. Je vois tous les chemins du ciel, les points qui brillent plus forts, comme des balises. Je vois les pistes secrètes, les puits sombres, les pièges. Je pense au Corsaire inconnu, qui a dormi peut-être sur cette grève, il y a si longtemps. Peut-être a-t-il connu ce vieux tamarinier qui gît maintenant sous la terre? N'a-t-il pas regardé avidement ce ciel qui l'avait guidé jusqu'à l'île? Allongé sur la terre douce, après la violence des combats, les meurtres, c'est ici qu'il a goûté la paix et le repos, abrité du vent de la mer par les cocotiers et les hyophorbes. J'ai franchi le temps, dans un vertige, en regardant le ciel étoilé. Le Corsaire inconnu est ici même, il respire en moi, et c'est avec son regard que je contemple le ciel (ibid.: 333).

Le mouvement est un principe vital. Dans L'Inconnu sur la terre, le ciel est animé par la migration des oiseaux, le passage de cerfs-volants, de lanternes, de nuages, d'orages. Notons que la seule vidéo réalisée par le jeune Le Clézio se cantonnait à filmer le passage de nuages. Le mouvement semble soulager le narrateur: "Le bleu du ciel, insistant, inamovible - lumière tendue - et l'apaisement, quand viennent les premiers nuages" (Le Clézio 1978: 103). Alexis est aussi sensible au mouvement céleste "[ $[.$.$] et les étoiles girent lente-$ ment jusqu’à l'aube" (Le Clézio 1985: 245). Et il se meut à son tour: "Je vais vers l'espace, vers l'inconnu, je glisse au milieu du ciel, vers une fin que je ne connais pas” (ibid.: 142). Ces deux extraits tendent le personnage entre l'aube, et sa vertu de commencement, et une fin, peut-être sans finitude.

Enfin, la troisième manière de mouvoir, déjà perceptible dans le précédent extrait, concerne la sensation, la possibilité d'émouvoir l'immobile: "Alors immobile, je ressens toutes les pulsations qui viennent, je les sens comme la circulation d'un sang essentiel, et le courant de l'air qui souffle en moi et me purifie comme un grand vent" (1978: 133). Bien que cette phrase commence par une stase, elle décline l'isotopie de la pneuma, dans une rêverie animée et une expression sensible qui permet de "partager non pas une identité, mais un même souffle (pneuma)” (Coccia 2016: 71). Faut-il rappeler qu'Adam, le premier protagoniste leclézien, signifie souffle? 
S'émanciper du primat de la vue suppose une expérience phénoménologique, un flottement qui fait, comme l'écrit Merleau-Ponty, que l'"être perceptif amputé de son monde dessine une spatialité sans choses” (Merleau-Ponty 1945: 328). Parmi les multiples passages du Chercheur d'or où le personnage contemple le ciel, le passage suivant marque bien la tension entre mouvement et immobilité puis comment la sensation corporelle et l'hallucination auditive prennent le pas sur la vue:

C'est une nuit de lune noire, comme disait mon père autrefois. Les étoiles emplissent le ciel, et je les contemple, pris par cette folie. Je parle tout haut, je dis: je vois le dessin, il est là, je le vois. Le plan du Corsaire inconnu n'est autre que le dessin de la Croix du Sud et de ses "suiveuses", les "belles de nuit". Sur l'étendue immense de la vallée, je vois briller les pierres de lave. Elles sont allumées comme des étoiles dans l'ombre poussiéreuse. Je marche vers elles, les yeux agrandis, je sens sur mon visage la braise de leurs lumières. La soif, la faim, la solitude tourbillonnent en moi, de plus en plus vite. J'entends une voix qui parle, avec les intonations de mon père. Cela me rassure d'abord puis me fait frissonner, car je m’aperçois que c'est moi qui parle. Pour ne pas tomber, je m'assois sur la terre, près du grand tamarinier qui m'abrite le jour. Le frisson continue ses vagues sur mon corps, je sens entrer en moi le froid de la terre et de l'espace. (Le Clézio 1985: 204-205)

Il s'agirait moins de relier l'aérien et le chtonien que de concevoir une unité atmosphérique. Ce changement de paradigme suppose de cesser de se placer au-devant, face, ou en surplomb pour, au contraire, en être dans un rapport plus inclusif. Le philosophe Emanuele Coccia a forgé le terme de plasmabilité pour désigner "Un monde où action et contemplation ne se distinguent plus",

un monde où matière et sensibilité - ou, si l'on veut, œil et lumière - s'amalgament parfaitement. Corps et organes de sensibilité ne peuvent plus être séparés. Nous ne sentirions plus avec une seule partie de notre être, mais avec la totalité de notre être. Nous ne serions qu'un immense organe de sens qui se confond avec l'objet perçu. Une oreille qui n'est que le son qu'elle entend, un œil qui baigne constamment dans la lumière qui lui donne vie. (Coccia 2016: 49)

L'expérience de perméabilité requiert d'être un seul organe qui suppose une atmosphère échappant à la partition terre/ciel. Cette conception déboucherait sur une éthique de la non-propriété. Néanmoins, quitter le rapport exclusif et dominant n'est pas si simple, que l'on se souvienne de l'énoncé dans Le Procès-verbal sur l'esprit de conquête spatiale dans un enjeu de domination:

Les journaux disent: "Le vaisseau spatial Liberté II a tourné sept fois autour de la terre." "La Bombe H de 100 mégatonnes a explosé dans le Nevada". C'était en effet comme si un gros soleil avait lui partout, tout le temps. Un soleil en forme de poire, mesurable en degrés Beaufort, un soleil à aurores démultipliées. On était en train de tresser un réseau inextricable autour de la planète. On la quadrillait méthodiquement, en prolongeant des lignes xx', yy', zz'. Et en contrôlait chaque carré. (Le Clézio 1963: 181-182)

Les savoirs occidentaux ont un tropisme: le géocentrisme et la géométrie l'attestent. “[N]ous n'avons jamais été réellement héliocentriques”. La terre est La mesure par rapport à laquelle sont définis mouvement et repos, dans une conception plus husserlienne 
que copernicienne. Or la terre n'est pas autonome par rapport au soleil, et elle n'est qu'un astre parmi d'autres. Et, selon Coccia, tout ce qui se vit sur terre "est de nature astrale", "Il n'y a que du ciel, partout, et la terre en est une portion, un état d'agrégation partielle" (Coccia 2016: 117). "L’enjeu pour Copernic n'était pas simplement l'affirmation de la centralité du Soleil. Poser le Soleil au milieu de tout c'est accomplir plusieurs déplacements cognitifs et métaphysiques. Postuler qu'au centre de l'Univers il y a le Soleil signifie, tout d'abord, universaliser le mouvement. La Terre a besoin de tourner autour du Soleil pour pouvoir exister: toute sa réalité doit être comprise et observée à partir de cette source infinie de lumière et d'énergie" (Coccia 2016: 118) De ce paradigme astral, il s'ensuit une manière d'être au monde, qui passe par la non-propriété, pour accepter l'impossible cielitoire ${ }^{3}$, car le ciel est infini, "sans frontières" (Le Clézio 1978: 293). "Reconnaître que le monde est un espace d'immersion signifie, [... ] qu'il n'a pas de frontières stables ou réelles: le monde est l'espace qui ne se laisse jamais réduire à une maison, au propre, au chez-soi, à l'immédiat" (Le Clézio 1978: 61).

Emanuele Coccia pense le souffle et l'existence fondamentalement pneumatologique: "On ne peut que respirer le monde" (Coccia 2016: 77). Et il nous invite à considérer l'environnement autrement qu'en termes d'habitat, pour parvenir à se sentir dans un tout. Le Clézio joue de cette tension ciel/terre et nous conduit à réfléchir à notre manière d'habiter (Bouvet [\&] Colin 2017), et nul doute qu'il valorise une approche plus poético-anthropologique, en évoquant des "gens des nuages", qu'une conception de l'habiter dominante.

Nous avons vu comment il peut activer une expérience sensible du regard sans immobiliser artificiellement le ciel en une image. Dans Le Chercheur d'or, la contemplation rend le personnage sensible à la dilatation de la durée, à l'instant précis et, tout à la fois, à la mémoire d'un autre temps. Tandis que L'Inconnu sur la terre est un point de bascule, un récit blanc de lumière, ou un roman à blanc qui n'accouche pas de sa fable. Une telle extension de la lumière renverse l'aphorisme de René Char à savoir "La lumière a un âge, la nuit n'en a pas” (Char 1995: 379). Avec J.M.G. Le Clézio, la lumière n’a pas d'âge. La lumière anesthésie, aveugle tandis que la nuit étoilée est propice à la rêverie. Pour Alexis, entre ciel et soleil, l'aveuglement est réel et le mode opératoire du dé-cillement s'exerce à ses dépens.

Avant de conclure, faisons un détour par une fable, possible palimpseste, qui a connu de multiples adaptations depuis Esope. Dans la version de Socrate (de la Théétète de Platon): Thalès regarde le ciel et tombe dans un puits, la servante Thrace se moque: dans le désir de connaître ce qu'il y a dans le ciel, il ignorait ce qu'il y avait devant lui et sous ses pieds. Alexis a ce point commun avec Thalès de fixer le ciel, au point de ne pas saisir sa vie. Quand Bergson reprend la fable, il met l'accent sur l'intention: "Sans doute une chute est toujours une chute, mais autre chose est de se laisser choir dans un puits parce qu'on regardait n'importe où ailleurs, autre chose y tomber parce qu'on visait une étoile" (Bergson 1959: 391-393). À la lecture d'Emanuele Coccia, il n'y aurait plus ni visée ni vue de l'esprit. Comme chez le peuple maya, si cher à J.M.G. Le Clézio, il s'agit de pressentir que "ce monde terrestre n'est pas autonome, mais qu'il est une parcelle de l'infini, et que ce temps n'est qu'un passage sur la grande roue du temps" (Le Clézio 1976: 11).

Aussi au terme de plasmabilité, nous ajoutons celui d'illimitation, principe actif, bien moins arrêté que ne le suggère le terme d'illimité. Et on aura remarqué la fréquence chez

\footnotetext{
3 Néologisme de notre fait (Roussel-Gillet 2007: 152-163).
} 
Le Clézio de l'expression “qui n’a pas de fin" (Onitsha). De cette vitalité extensive témoigne L'Inconnu sur la terre. Et la répétition d'un livre à l'autre ne crée pas un simple faisceau d'intratextualité, elle inscrit aussi l'expérience dans un monde phénoménologique qui est fondamentalement intersubjectif comme l'écrit Maurice Merleau-Ponty,

non pas de l'être pur, mais le sens qui transparait à l'intersection de mes expériences et de celles d'autrui, par l'engrenage des unes sur les autres, il est donc inséparable de la subjectivité et de l'intersubjectivité qui font leur unité par la reprise de mes expériences passées dans mes expériences présentes, de l'expérience d'autrui dans la mienne. (Merleau-Ponty 2010: 671)

Dans Le Chercheur d'or, Alexis connecte ses expériences passées aux présentes et à celles d'autres figures - le père, le Corsaire. Et si l'univers céleste renvoie à l'infini, il correspond aussi à ce qui caractérise la chose littéraire, selon Maniglier "une certaine vibration d'être, une manière de se déconstruire et de se reconstruire sur place, de ne pas tenir dans ses propres limites, dans sa propre matière, dans sa réalité" (Maniglier 2013: 48-80).

Si regarder le ciel invite à la contemplation nostalgique ou romantique, et faire résonance entre passé et présent, il peut aussi s'agir de vivre l'expérience vitaliste, sans visée, d'éprouver l'air, élément moins analysé dans l'œuvre de Le Clézio. Entre destinée et destinement, l'individuation permet de rejoindre l'autre, qui peut être humain ou élément. Dans ces circonstances, le paradigme astral existe bien chez Le Clézio, la plasmabilité dans l'essai poétique L'Inconnu sur la terre a ouvert une brèche, dont on retrouve les sédiments dans les livres ultérieurs. Même si tout roman, tel Le Chercheur d'or fortement empreint de modèles, ne baigne pas entièrement dans cette plasmabilité, cette dernière est éprouvée, ne serait-ce que par intermittences. C'est alors une manière éthique de ne plus avoir de rapport d'habitat au monde à la manière occidentale, de le déshabiter, en quelque sorte, pour en être autrement.

\section{Bibliographie}

Bergson, Henri 1959. Le rire. Ouvres, chapitre I, 2. Paris: PUF.

Char, René 1995. Euvres complètes, Paris: Gallimard. La Pléiade.

Chung, Ook 2001. Le Clézio, une écriture prophétique. Paris: Imago.

Coccia, Emanuele 2016. La vie des plantes, métaphysique du mélange. Paris: Bibliothèque Rivages.

Collot, Michel 2005. La poésie moderne et la structure d'horizon. Paris: PUF.

Bouvet, Rachel [\&] Claire Colin (dirs.) 2017. Habiter la terre. Les Cahiers J.M.G. Le Clézio 10. Passage (s).

Descola, Philippe 2005. Par-delà nature et culture. Paris: Gallimard.

Didi-Huberman, Georges 2018. "Libres yeux de l'histoire". Europe, Georges Didi-Huberman, James Sacré 1069, 20 (mai): 18-30.

Heidegger, Martin 1990 [1976]. Questions III et IV. Paris: Gallimard, Tel.

Jung, Carl Gustav 2014 [1944]. Psychologie et alchimie. Paris: Libella.

Le Clézio, Jean-Marie Gustave 1963. Le Procès-verbal. Paris: Gallimard.

— 1976. Les Prophéties de Chilam Balam. Paris: Gallimard.

— 1978. L'inconnu sur la terre. Paris: Gallimard.

— 1985. Le Chercheur d'or. Paris: Gallimard. 
1991. Onitsha. Paris: Gallimard.

2000. Cour brûle et autres romances. Paris: Gallimard.

Maniglier, Patrice 2013. "Du mode d'existence des objets littéraires: enjeux philosophiques du formalisme". Les temps modernes 5 (676): 48-80.

Merleau-Ponty, Maurice 2010 [1945]. Phénoménologie de la perception. Paris: Gallimard.

Nancy, Jean-Luc 2003. Au fond des images. Paris: Galilée.

Roussel-Gillet, Isabelle 2007. “Le Clézio passeur au monde: l'Écriture et le passage des seuils”. Nouvelles Études Francophones 22, 2 (Automne): 152-163.

Roussel-Gillet, Isabelle 2008. "Les cartes du ciel à l'œuvre chez Le Clézio”. Textimage, Cartes et plans 2 (Eté). https://www.revue-textimage.com/03_cartes_plans/roussel.pdf [15.12.2018].

Waelti-Walters, Jennifer 1981. Icare ou l'évasion impossible, étude psycho-mythique de l'ouvre de J.M.G. Le Clézio. Québec: Namaan. 